\title{
In Situ Capture RT-qPCR Method for Detection of Human Norovirus in Food and Environmental Samples ${ }^{+}$
}

\author{
Peng Tian \\ Produce Safety \& Microbiology Research Unit, Western Reginal Research Center, ARS, USDA, \\ Albany, CA 94710, USA; peng.tian@usda.gov \\ † Presented at Viruses 2020-Novel Concepts in Virology, Barcelona, Spain, 5-7 February 2020. \\ Published: 1 June 2020
}

\begin{abstract}
Human noroviruses (HuNoVs) are the major cause of non-bacterial acute gastroenteritis worldwide. RT-qPCR is a widely used method to detect HuNoVs. However, the method is unable to extract a virus from environmental samples and to discriminate between infectious and noninfectious viruses. In this study, we explored a new in situ capture RT-qPCR (ISC-RT-qPCR) methodology to estimate the infectivity of $\mathrm{HuNoV}$ in environmental and food samples. This assay was based on capturing encapsidated $\mathrm{HuNoV}$ by viral receptors, followed by in situ amplification of the captured viral genomes by RT-qPCR. We demonstrated that ISC-RT-qPCR did not capture and enable signal amplification of the heat-denatured Tulane virus (TV) and HuNoVs. Therefore, ISC-RT-qPCR provides better estimates for infectivity of HuNoV than RT-qPCT. We then utilized the ISC-RT-qPCR to detect HuNoV in environmental water samples and food samples, as compared to a conventional RT-qPCR procedure. The presence of HuNoV was examined in 36 oyster samples from retail markets using by both assays for detection. The detection rates of HuNoV in gill, digestive glands, and other tissues were 33.3\%, 25\%, and 19.4\%, respectively, by ISC-RT-qPCR; and were $5.6 \%, 11.1 \%$, and $11.1 \%$, respectively, by RT-qPCR. ISC-RTqPCR is more sensitive than RTqPCR for the detection of $\mathrm{HuNoV}$ in oysters. By contrast, the HuNoV detection rate by ISC-RTqPCR is lower for environmental samples. Of the 72 water samples that tested positive for HuNoV by RTqPCR, only $20(27.8 \%)$ of these tested positive by ISC-RT-qPCR, suggesting that $72.2 \%$ of RT-qPCRpositive samples were unlikely to be infectious. A better detection rate by ISC-RT-qPCR in oyster samples indicates the likelihood of infectious HuNoV that accumulated in oysters, and a lower detection rate of $\mathrm{HuNoV}$ in environmental water by ISC-RT-qPCR, indicating that the majority of RT-qPCR-positive samples were from non-infectious viral RNA.
\end{abstract}

Keywords: HuNoV; ISC-RT-qPCR; detection; environmental samples 